\title{
Outbreak of Respiratory Tract Infection Score (ORTIS): Objective Screening System for Children to Rule out COVID-19 and Prevent Nosocomial Spread
}

\author{
Varnit Shanker $^{*}$ \\ Consultant Pediatrician and Neonatologist, DACH Jaipur, India
}

Since December 2019, over two million people worldwide have been affected by coronavirus disease 2019 (COVID-19). Because of quarantine measures, overcrowded healthcare facilities, fear of contracting the disease and forced behavioural modification to suspect every respiratory tract infection as a potential COVID-19 case - parents of sick children are hesitant to contact healthcare facilities in developing countries such as India. Acute shortage of specialist care has led to increased suffering of children affected by other diseases. Such patients are being continuously received in critical state due to a delay in contact. In resource scarce settings with an incomplete understanding of the disease process, there is also a lack of confidence amongst pediatricians while screening the patients for COVID-19 clinically. This problem has compounded due to infected doctors and staff who constitute the first and only line of defence against the pandemic, leading to sub-optimal levels of functioning of many healthcare facilities.

Haiyan Qiu and colleagues elaborated little known clinical and epidemiological details of COVID-19 in pediatric population [1]. To help pediatricians clinically screen the patients of COVID-19 and comfortably deal with patient families with unlikely coronavirus infection, a scoring system called "Outbreak of Respiratory Tract Infection Score" or ORTIS System has been devised (Table 1). ORTIS was conceptualized based on expert opinion and review of literature of past outbreaks of viral infections in children with predominant respiratory system involvement [1-5]. The primary purpose of the scale is to confidently rule out the chances of CoVID-19 infection.

The scale was tested by screening 93 consecutive children in the outpatient department of a tertiary care pediatric hospital in North India, between 14 March and 25 March, 2020 (Figure 1). No risk (24, 25.8\%), Low risk (31, $33.3 \%)$, Medium risk $(22,23.6 \%)$ and High risk (16, $17.2 \%)$ children were screened using ORTIS System. Hospital administrative staff, nursing staff and pediatric residents reported a higher degree of comfort and confidence when patients were labelled as No or Low risk. Most (14/22) medium risk patients were given telephonic instructions regarding symptomatic treatment. 8 out of 22 medium risk patient were called for examination and only 2 of the 22 patients required hospital admission. Not all High risk pa- tients were referred to COVID-19 facility, but all of them were asked to exercise extreme caution and were only given telephonic consultations. All 55 "No and Low risk" cases recovered clinically, they were found to be asymptomatic 7 days after consultation. Based on clinical judgement, all these cases were considered true negatives and not asymptomatic carriers. Hence, ORTIS system showed a $100 \%$ Negative Predictive Value.

ORTIS has been designed for implementation in ongoing and any future viral outbreaks with respiratory failure or pneumonia as possible complications. The ORTIS System can be performed telephonically which is critical in bifurcating the suspected patients towards COVID-19 dedicated hospitals during the early stages of clinical presentation. This step is crucial in reducing the risk of nosocomial transmission, infection transmission to the community and to the healthcare workers. "No risk" children are typically patients with straightforward diagnosis such as UTI or Neonatal Septicemia. "Low risk" children can be assumed to have an unlikely risk of COVID-19 infection, "Medium risk" children should be carefully evaluated and a follow up evaluation is a must. "High risk" cases should be urgently referred to dedicated centres for outbreak disease. The pilot study has important limitations such as low sample size and study participants not tested for SARS-CoV-2 infection by PCR analysis for microbiological confirmation. Although inter observer variation was not assessed, the objective and history based construct of scoring minimizes chances of any variations.

*Corresponding author: Varnit Shanker, MBBS, DCH, MRCPCH, HES-ALM Candidate, Consultant Pediatrician and Neonatologist, DACH Jaipur, Director, Center for Active Research Diagnostics and Infection Surveillance (CARDIS), India, Tel: +91-9929779306

Received: April 09, 2020

Accepted: April 27, 2020

Published online: April 30, 2020

Citation: Shanker V (2020) Outbreak of Respiratory Tract Infection Score (ORTIS): Objective Screening System for Children to Rule out COVID-19 and Prevent Nosocomial Spread. Ann Microbiol Res 4(1):102-104 
Citation: Shanker V (2020) Outbreak of Respiratory Tract Infection Score (ORTIS): Objective Screening System for Children to Rule out COVID-19 and Prevent Nosocomial Spread. Ann Microbiol Res 4(1):102-104

Table 1: Outbreak of Respiratory Tract Infection Scale (ORTIS System).

\begin{tabular}{|c|c|c|c|}
\hline SN & \multicolumn{2}{|l|}{ Clinical Screening Criteria } & Score \\
\hline 1 & \multicolumn{2}{|c|}{$\begin{array}{l}\text { History of contact with positive CoVID-19 case or a suspected CoVID-19 case or from localities where CoVID-19 cases have } \\
\text { been documented }\end{array}$} & 10 \\
\hline 2 & \multicolumn{2}{|l|}{ History of travel out of city / town / village } & 8 \\
\hline 3 & \multicolumn{2}{|l|}{ Radiological finding on Chest X-Ray suggesting Pneumonia } & 5 \\
\hline 4 & \multicolumn{2}{|l|}{ Loss of Taste or Loss of Smell or Both } & 5 \\
\hline 5 & \multicolumn{2}{|l|}{ Breathlessness / SpO2 <95\% } & 3 \\
\hline 6 & \multicolumn{2}{|c|}{ Fast Breathing / Tachypnea in absence of history of wheezing or asthma } & 2 \\
\hline 7 & \multicolumn{2}{|l|}{ Fever } & 2 \\
\hline 8 & \multicolumn{2}{|l|}{ Dry Cough } & 1 \\
\hline 9 & \multicolumn{2}{|l|}{ Throat congestion } & 1 \\
\hline 10 & \multicolumn{2}{|l|}{ Cough with sputum or Coryza or Both } & 1 \\
\hline 11 & \multicolumn{2}{|l|}{ Headache or Myalgia or Both } & 1 \\
\hline 12 & \multicolumn{2}{|l|}{ Loose stools / Vomiting / Pain Abdomen / Nausea } & 1 \\
\hline 13 & \multicolumn{2}{|l|}{ Unexplained Leucopenia or Thrombocytopenia } & 1 \\
\hline 14 & \multicolumn{2}{|l|}{ Any urinary Symptoms } & 0 \\
\hline 15 & \multicolumn{2}{|l|}{ Epistaxis } & 0 \\
\hline 16 & \multicolumn{2}{|c|}{ Night time and Early morning Coughing and History of Asthma / Recurrent wheezer } & 0 \\
\hline 17 & \multicolumn{2}{|c|}{$\begin{array}{l}\text { Neonate } \\
\text { (mother not CoVID-19 positive, no History of fever and cough) }\end{array}$} & 0 \\
\hline 18 & \multicolumn{2}{|l|}{ Febrile Seizures / Neck Rigidity and Fever and Vomiting } & 0 \\
\hline 19 & \multicolumn{2}{|c|}{$\begin{array}{l}\text { Follow up visit for chronic condition such as Diabetes, Nephrotic Syndrome, Seizure Disorder, etc. and planned } \\
\text { Immunization visits }\end{array}$} & 0 \\
\hline 20 & \multicolumn{2}{|l|}{ Trauma } & 0 \\
\hline & Total Score & Interpretation & \\
\hline & 0 & No Risk & \\
\hline & $1-2$ & Low Risk & \\
\hline & $3-9$ & Medium Risk & \\
\hline & 10 or More & High Risk & \\
\hline
\end{tabular}

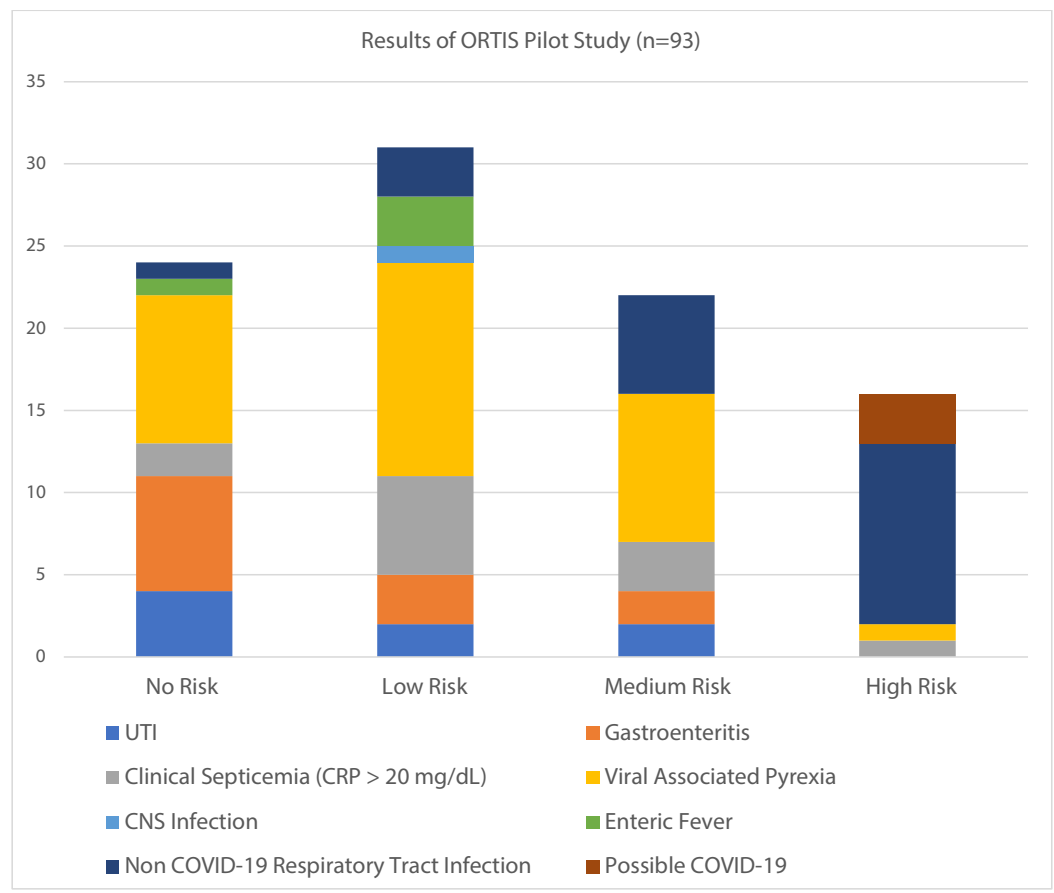

Figure 1: Results of Pilot Study, patient stratification across various ORTIS System risk zones. 


\section{Author's Contribution}

VS was responsible for conception of idea, literature review, preparation of manuscript and building figures and tables.

\section{Conflict of Interest}

None.

\section{Funding}

Not Applicable.

\section{References}

1. Qiu H, Wu J, Hong L, et al. (2020) Clinical and epidemiolog- ical features of 36 children with coronavirus disease 2019 (COVID-19) in Zhejiang, China: An observational cohort study. Lancet Infect Dis.

2. Dong $\mathrm{Y}, \mathrm{Mo} \mathrm{X}, \mathrm{Hu} \mathrm{Y}$, et al. (2020) Epidemiological characteristics of 2143 pediatric patients with 2019 coronavirus disease in China. Pediatrics.

3. Ogimi C, Englund JA, Bradford MC, et al. (2019) Characteristics and outcomes of coronavirus infection in children: The role of viral factors and an immunocompromised state. J Pediatric Infect Dis Soc 8: 21-28.

4. Stockman LJ, Massoudi MS, Helfand R, et al. (2007) Severe acute respiratory syndrome in children. Pediatr Infect Dis J 26: 68-74.

5. Memish ZA, Perlman S, Kerkhove MDV, et al. (2020) Middle East respiratory syndrome. Lancet 395: 1063-1077. 